\title{
Susceptibilidad in vitro de bacterias anaeróbicas en infecciones pleuropulmonares
}

\author{
Tamara Soler V, Lucía Salamanca F, Eliana Molinaa. \\ In vitro antimicrobial susceptibility \\ of anaerobic bacteria isolated \\ from pleuropulmonary infections
}

Background: Aspirative pleuropulmonary infections are usually caused by anaerobic flora of the mouth, mainly Prevotella, Fusobacterium and Peptostreptococcus spp. Penicillin in high doses is the traditional treatment for this type of infections but the rising resistance developed in recent years has induced the empiric use of clindamycin, increasing treatment costs. Aim: To study antimicrobial susceptibility of anaerobic bacteria isolated from pleuropulmonary infections. Material and methods: Thirty two strains obtained from bronchoalveolar lavage and 15 strains isolated from pleural effusions between 2000 and 2002, were studied. The phenotype of strains was identified using the semiautomated API 20 A method and their susceptibility to penicillin (PNC), clindamycin (CM) and chloramphenicol (CAF) was tested using the E test methods. Results: All the strains were susceptible to CAF, $95 \%$ to CM and $74.4 \%$ to PNC. The predominant genus was Prevotella, which also exhibited the higher resistance. Conclusions: As CM and CAF are active "in vitro", high rates of clinical response should be expected. In contrast, PNC is less effective, especially against pigmented Prevotella (Rev Méd Chile 2006; 134: 465-8).

(Key words: Anti-bacterial agents; bacteria, anaerobic; Drug-resistance, microbial; Prevotella)

Recibido el 6 de agosto, 2004. Aceptado el 12 de septiembre, 2005.

Trabajo financiado con aporte parcial de Laboratorios Pfizer.

Servicio de Medicina, Laboratorio de Bacteriología, Instituto Nacional del Tórax. Santiago de Chile.

aTecnólogo Médico

$E^{1}$ estudio de sensibilidad de bacterias anaerobias no se realiza rutinariamente, ya que existen dificultades tanto en la obtención de muestras no contaminadas con la flora del propio paciente, como en el cultivo, ya que estas bacte-

Correspondencia a: Dra. Tamara Soler. Servicio de Medicina Instituto Nacional del Tórax. rias son de muy lento crecimiento y requieren de condiciones especiales de anaerobiosis, lo que implica mayor dificultad y $\operatorname{costos}^{1}$.

En Chile se publicó un estudio de sensibilidad de anaerobios en 1981, donde la penicilina demostraba variada actividad, con mala respuesta de algunos bacilos Gram negativos. La mayor sensibilidad en los anaerobios se presentó con cloramfenicol y metronidazol, no se evaluó clindamicina ${ }^{2}$. 
La penicilina $\mathrm{G}$ siempre fue la primera alternativa del tratamiento de infecciones por anaerobios por sobre el diafragma, sin embargo, en los últimos años han aparecido cepas resistentes, especialmente de Prevotella melaninogenica (antes Bacteroides melaninogenicus) que son resistentes hasta en $20 \%$ de los $\operatorname{casos}^{3,4}$.

En una revisión previa en nuestro Servicio se detectaron enfermos con procesos anaeróbicos con mala respuesta clínica al tratamiento antimicrobiano de penicilina en dosis altas, requiriendo cambio de terapia a clindamicina o cloramfenicol, comprobándose en la revisión de cultivos que un alto porcentaje de estos casos tenían bacilos Gram negativo pigmentados correspondientes al Prevotella ${ }^{5}$.

Es importante conocer la sensibilidad en nuestro medio y los cambios a través del tiempo, para poder indicar una terapia antimicrobiana que en la mayoría de estas infecciones es empírica por las dificultades de los cultivos antes mencionadas.

El objetivo del presente trabajo es determinar la susceptibilidad in vitro de cepas respiratorias anaerobias a penicilina G (PNC), cloramfenicol (CAF) y a clindamicina $(\mathrm{CM})$ y conocer las especies más relevantes causantes de infecciones pleuropulmonares en pacientes hospitalizados en nuestro servicio.

\section{Material y MÉtodos}

Entre los años 2000 y 2002 se analizaron muestras obtenidas por fibrobroncoscopia o por punción pulmonar de pacientes con patología pleuro-pulmonar en nuestro hospital, en búsqueda de agentes anaerobios. Se cultivaron en agar sangre anaerobio adicionado de amikacina, en anaerobiosis en jarra Gaspak a $37^{\circ} \mathrm{C}$ entre $48 \mathrm{~h}$ y seis días.

Se aislaron 47 cepas anaeróbicas de 38 pacientes, las que se identificaron con el método semiautomatizado API 20 A (BioMérieux) con incubación en anaerobiosis por 24 a $72 \mathrm{~h}$.

Las muestras estudiadas correspondieron a 32 lavados broncoalveolares (LBA) (68\%), con recuentos cuantitativos altos ( $\left.>10^{5} \mathrm{UFC} / \mathrm{ml}\right), 12$ líquidos pleurales $(25,6 \%)$ y 3 pus de absceso $(6,3 \%)$.

Se determinó la concentración inhibitoria mínima (CIM) con tiras con gradiente de concentración de antimicrobiano E Test (AB Biodisk) por el método de difusión en agar Brucella - sangre de cordero al $5 \%$. Se colocaron dos tiras por placa y se incubaron a $37^{\circ} \mathrm{C}$ por 24 a $48 \mathrm{~h}$. Se utilizaron tiras de penicilina $\mathrm{G}$ en 45 cepas, clindamicina (en 44 cepas) y cloramfenicol en 44 cepas.

Los niveles de corte de CIM se aplicaron según las normas del National Committee for Clinical Laboratory Standards (NCCLS) (Center for Diseases Control (CDC), USA) 1995; M11-A3 (Esp.) ${ }^{1}$.

\section{Resultados}

De 45 cepas estudiadas frente a penicilina 33 fueron sensibles $(73,3 \%), 4$ con sensibilidad intermedia $(8,9 \%)$ y 8 resistentes (17,8\%). De 44 cepas enfrentadas a clindamicina, 42 fueron sensibles $(95,5 \%)$ y sólo $2(4,5 \%)$ resistentes. De 44 cepas estudiadas con cloramfenicol, todas fueron sensibles (100\%) (Tabla 1). Todas las cepas sensibles a PNC fueron sensibles a cloramfenicol y clindamicina.

Se estudiaron 31 bacilos Gram negativo (BGN), de los cuales 19 presentaron pigmento café a negro (cepas pigmentadas) y 12 fueron no pigmentadas. Entre las 19 cepas oscuras, 14 fueron Prevotella spp (con predominio de P melaninogenica/oralis con 6 cepas) y 2 Porphyromonas sp; 3 no se identificaron. De las 12 cepas de BGN no pigmentadas, 5 fueron Bacteroides spp, 2 Fusobacterium spp (necrophorum 0 nucleatum), 1 Prevotella oris/buccae, 1 Porphyromonas sp y 3 no pudieron ser identificadas. De 12 bacilos Gram positivo (BGP), 11 se identificaron, con predominio de Propionibacterium acnes con 5 cepas, 3 Propionibacterium sp, 2 Clostridium sp y 1 Bifidobacterium sp). De 4 cepas de cocos Gram positivo (CGP) 3 correspondieron a microaerófilos que sólo se aislaron en ambiente anaerobio. Una de ellas, Gemella, sólo se logró estudiar con penicilina y fue resistente.

Se determinó la CIM de PNC en 29 cepas de BGN, siendo sensibles $62 \%$ (n 18), resistentes $27,6 \%$ $(n=8)$ y con sensibilidad intermedia $10,4 \%(n=3)$.

Relacionando la resistencia con la presencia de pigmento, de 18 cepas pigmentadas estudiadas con penicilina, 10 presentaron resistencia o sensibilidad intermedia. Al contrario, 10 de las 11 cepas no pigmentadas fueron sensibles.

El género bacteriano con mayor resistencia fue Prevotella: de 15 cepas sólo 7 fueron sensibles a 


\section{Tabla 1. Susceptibilidad de 47 cepas anaeróbicas aisladas del tracto respiratorio bajo Int 2000-2002}

\begin{tabular}{|c|c|c|c|c|c|c|}
\hline \multirow[t]{2}{*}{ Susceptibilidad } & \multicolumn{4}{|c|}{$\begin{array}{c}\text { Antimicrobiano } \\
\text { Cloramfenicol } \\
n=44\end{array}$} & \multicolumn{2}{|c|}{$\begin{array}{c}\text { Penicilina G } \\
\mathrm{n}=45\end{array}$} \\
\hline & $\mathrm{n}$ & $\%$ & $\mathrm{n}$ & $\%$ & $\mathrm{n}$ & $\%$ \\
\hline Sensibles & 42 & 95,5 & 44 & 100,0 & 33 & 73,3 \\
\hline Sensibil. Interm. & 0 & 0 & 0 & 0 & 4 & 8,9 \\
\hline Resistentes & 2 & 4,5 & 0 & 0 & 8 & 17,8 \\
\hline
\end{tabular}

penicilina, 6 resistentes y 2 con sensibilidad intermedia. De 2 cepas pigmentadas de Porphyromonas spp 1 fue resistente. Dos cepas sin pigmento de Porphyromonas y Prevotella fueron sensibles a penicilina.

Hubo solamente 2 cepas resistentes a clindamicina, que correspondieron a Prevotella melaninogenica/oralis (colonia pigmentada) y a Gemella morbilorum (CGP) (microaerófilo sin pigmento).

Las 12 cepas de bacilos Gram positivo fueron sensibles a los 3 antimicrobianos.

Al comparar la susceptibilidad de las 12 cepas resistentes e intermedias a penicilina con los otros 2 antimicrobianos, se observó que sólo 2 fueron resistentes a clindamicina y todas fueron sensibles a cloramfenicol (Tabla 2). Casi todas eran pigmentadas.

\section{DisCUSIÓN}

Los gérmenes más frecuentemente involucrados en las neumonías o procesos pulmonares por anaerobios corresponden a aquéllos que colonizan la orofaringe: cocos Gram positivo y bacilos Gram negativo como: Prevotella melaninogenica, Bacteroides spp, Fusobacterium spp y Peptostreptococcus spp.

Los estudios de susceptibilidad rutinarios para estos gérmenes anaerobios no son recomendados básicamente por dos razones. La primera incluye las dificultades técnicas en obtener muestras representativas, especialmente de la vía aérea, ya que los métodos principales, como las punciones

\section{Tabla 2. Susceptibilidad (CIM) a clindamicina y cloramfenicol de cepas resistentes e intermedias a penicilina $\mathbf{G}$ y producción de pigmento Int 2000 - 2002}

\begin{tabular}{|lcrrrrrr|}
\hline Cepa & Pigmento & \multicolumn{2}{c}{ Clindamicina } & \multicolumn{2}{c|}{ Cloramfenicol } & \multicolumn{2}{c|}{ Penicilina } \\
\hline Prevotella sp & + & $<0,016$ & $\mathrm{~S}$ & 0,75 & $\mathrm{~S}$ & 8,0 & $\mathrm{R}$ \\
Prevotella sp & + & - & & - & & 1,0 & $\mathrm{I}$ \\
Prevotella sp & + & $<0,016$ & $\mathrm{~S}$ & $<0,016$ & $\mathrm{~S}$ & 3,0 & $\mathrm{R}$ \\
Prev.melani/oralis & + & 0,125 & $\mathrm{~S}$ & 2,0 & $\mathrm{~S}$ & 1,5 & $\mathrm{I}$ \\
Prev.melani/oralis & + & 0,023 & $\mathrm{~S}$ & 0,5 & $\mathrm{~S}$ & 2,0 & $\mathrm{R}$ \\
Prev.melani/oralis & + & 0,016 & $\mathrm{~S}$ & 0,35 & $\mathrm{~S}$ & 2,0 & $\mathrm{R}$ \\
Prev.melani/oralis & + & $>256$ & $\mathrm{R}$ & 2,0 & $\mathrm{~S}$ & 6,0 & $\mathrm{R}$ \\
Prev.melani/oralis & + & 0,016 & $\mathrm{~S}$ & 0,5 & $\mathrm{~S}$ & 12,0 & $\mathrm{R}$ \\
Porphyromonas & & & & & & & \\
asaccharolytica & + & $<0,016$ & $\mathrm{~S}$ & $>0,016$ & $\mathrm{~S}$ & 3,0 & $\mathrm{R}$ \\
Bac.Gr.neg. s/identif. & + & $<0,016$ & $\mathrm{~S}$ & 0,23 & $\mathrm{~S}$ & 0,75 & $\mathrm{I}$ \\
Fusob.necro.nucleatum & $\mathrm{S} /$ pigmen. & 0,047 & $\mathrm{~S}$ & 0,016 & $\mathrm{~S}$ & 1,0 & $\mathrm{I}$ \\
Gemella morbilorum & $\mathrm{s} /$ pigmen. & 32,0 & $\mathrm{R}$ & 0,75 & $\mathrm{~S}$ & 4,0 & $\mathrm{R}$ \\
\hline
\end{tabular}

$\mathrm{CIM}=$ concentración inhibitoria mínima (en $\mathrm{mcg} / \mathrm{ml}$ ). $\mathrm{S}=$ sensible. $\mathrm{I}=$ intermedio. $\mathrm{R}=$ resistente. 
transtorácicas y transtraqueales, son invasivos. El lavado broncoalveolar con recuento cuantitativo, en nuestro medio se ha descrito como comparable a las muestras obtenidas por catéter protegido, por lo que la hemos considerado una muestra adecuada $^{6}$. La segunda dificultad es el difícil cultivo de los anaerobios que requieren medios especiales y de mayor costo tanto para su crecimiento como para la identificación 7,8 .

La elección de los antimicrobianos, para el tratamiento de infecciones pulmonares por anaerobios, se realiza en base al conocimiento epidemiológico de estas infecciones y a escasos estudios de susceptibilidad in vitro. Hasta hace unos años, la penicilina $\mathrm{G}$ se mantenía como la droga de elección. Sin embargo en los últimos años con la aparición de cepas de Prevotella (ex Bacteroides) melaninogenica que tienen hasta $25 \%$ de resistencia a este antimicrobiano, ha obligado a considerar otras alternativas terapéuticas en el tratamiento de infecciones pulmonares de este tipo ${ }^{9-11}$.

En este trabajo se encontró una sensibilidad de $100 \%$ de las cepas frente a cloramfenicol y $95,5 \%$ a clindamicina, mientras la penicilina mostró alrededor de $18 \%$ de cepas resistentes y $9 \%$ de cepas con sensibilidad intermedia o disminuida. Estos hallazgos son comparables a los descritos en la literatura.

\section{REFERENCIAS}

1. Vilinanova PA. Approved Standard, M11-A2 (Esp.): Methods for Antimicrobial Susceptibility Testing of Anaerobic Bacteria. National Committee for Clinical Laboratory Standards, 1995.

2. Valenzuela ME, SANhuEza A. Estudio de sensibilidad antibiótica de bacterias aisladas en Chile. Bol ISP 1981; 22: 45-9.

3. Murria PR, Rosenblatt JE. Penicillin resistance and penicillinase production in clinical isolates of Bacteroides melaninogenicus. Antimicrob Agents Chemother 1977; 11: 605-8.

4. Edson RS, Rosenblatt JE, Lee DT, Mc Vey RA III. Recent experience with antimicrobial susceptibility of anaerobic bacteria: increasing resistance to penicillin. Mayo Clinic Proc 1982; 57: 737-41.

5. Soler T, Barrientos P, Salamanca L. Infecciones pleuropulmonares por Bacteroides melaninogenicus. Revista Enf Res Cir Torác 1990; 6: 185-90.
Se observó mayor resistencia en los bacilos Gram negativo (como Prevotella y Bacteroides). Al igual que en otras series los anaerobios con mayor resistencia a la penicilina fueron del género Prevotella especialmente las cepas pigmentadas en las que encontramos más de $50 \%$ resistentes.

Los bacilos Gram positivo anaerobios fueron todos sensibles a la penicilina, clindamicina y CAF, por lo que no parecen presentar problemas serios en su tratamiento.

Frente a estos resultados, pensamos que la penicilina sigue siendo la droga de elección para comenzar el tratamiento de patologías respiratorias por anaerobios en nuestro país por su bajo costo y por tratarse de cuadros de evolución lenta que permiten observar la respuesta. Sin embargo, si ésta no es adecuada y en los cultivos anaeróbicos a los 5 o más días aparecen colonias pigmentadas, se justifica cambiar el antimicrobiano por la alta posibilidad de que sea resistente a penicilina, aun sin identificación ni estudio de sensibilidad.

En conclusión creemos que es importante realizar estudios de sensibilidad con fines de investigación para determinar la realidad local y facilitar las decisiones terapéuticas empíricas en las diferentes regiones geográficas.

6. Moreno R, Lobos T, Ovale J, Beroiza MT, Día O, Muñoz $\mathrm{V}$ ET AL. Comelación entre el cultivo de aerobios y anaerobios de lavado broncoalveolary catéter telescópico protegido. Rev Chil Enf Respir 1993; 9: 163-73.

7. Bartlett JG, O'Keefe P, Taluy FP, Louis TJ, Gorbach SI. Bacteriology of Hospital Acquired Pneumonia. Arch Intern Medicine 1986; 146: 868-71.

8. Scannapieco FA, Papandonate GD, Dunford. Association between oral contamination and respiratory disease in a National Sample Survey Population. Ann Periodontal 1998; 3: 251-6.

9. PerLino CA. Metronidazol vs clindamicina treatment of anaerobic pulmonary infection, failure of metronidazol therapy. Arch Intern Med 1981; 141: 1424-7.

10. BartLett JG. Aspiration pneumonia. In: Barm GL, Wolinsky E, edts. Textbook of Pulmonary Diseases. $5^{\text {th }}$ Ed New York. Little Brown, 1994; 593-606.

11. Неснт DW, Osmolski JR. Activities of Garenoxacin and other Agents Against Anaerobic Clinical Isolates. Antimicrob. Ag Chemother 2003; 47: 910-6. 